\title{
Integrative Levels of Program Comprehension
}

\author{
Reinhard Schauer* \\ Université de Montréal \\ Montréal, Canada \\ reinhard.schauer@kodak.com
}

\author{
Rudolf K. Keller ${ }^{\dagger}$ \\ PMOD Technologies \\ Zürich, Switzerland \\ keller@pmod.com
}

\begin{abstract}
Integrative levels of program comprehension describe the evolution of our knowledge about a program from low-level program elements through successive and higher orders of knowledge integration. In this paper, we motivate, define, and illustrate integrative levels as a technique to organize the knowledge that we acquire in the course of program maintenance. We differentiate between understanding in extension and understanding in intension. We define five intensional integrative levels of program comprehension, the element level, the percept level, the concept level, the subject level, and the domain level. The intensional units that make up these levels emerge and evolve in an iterative comprehension process that is active at each level. The iterations of this process comprise the four phases of lower level unit organization, unit integration, upper level unit organization, and unit validation. We demonstrate integrative levels of program comprehension using the object-oriented testing framework jUnit as a case study.
\end{abstract}

\section{Introduction}

Max Wertheimer analyzed the research approach that drove Galileo to the discovery of the laws of inertia, and he investigated in personal interviews the thought process that led Albert Einstein to the theory of general relativity. Wertheimer concluded that two directions were involved: "getting a whole consistent picture and seeing what the structure of the whole requires for the parts" [17]. In essence, this is what would become the official position of Gestalt theory:

\footnotetext{
* The first author carried out this research during a leave of absence from Kodak Graphic Communications Canada Company where he works as a Senior Software Developer.

${ }^{\dagger}$ The second author carried out part of this work as an Adjunct Professor at Université de Montréal, Canada.
}

\begin{abstract}
"Experiences are usually organized wholes whose parts are coordinated in a hierarchical system around a central point. Such structures are in no way less immediate than their parts; indeed one often apprehends a whole before anything regarding its parts is apprehended." [8]
\end{abstract}

In the decades thereafter, almost all disciplines of science debated the Gestalt theorists' moderate holistic stance on scientific analysis [18]. In particular, biology succeeded in explaining complex phenomena of nature by building a theory of comprehension around the notion of integrative levels. Many attributed this idea to Needham, who argued that the universe be governed at different levels of organization, by "successive forms of order in a scale of complexity and organization" [12]. Integrative levels were explored exhaustively in biology [13], philosophy [5], psychology [16], and physics [2]. Outstanding of all is Novikoff's distinct explanation:

"In the continual evolution of matter, new levels of complexity are superimposed on the individual units by the organization and integration of these units into a single system. What were wholes on one level become parts on a higher one. Each level of organization possesses unique properties of structure and behavior which, though dependent on the properties of the constituent elements, appear only when these elements are combined in the new system. Knowledge of the laws of the lower level is necessary for a full understanding of the higher level; yet the unique properties of phenomena at the higher level can not be predicted, a priori, from the laws of the lower level. The laws describing the unique properties of each level are qualitatively distinct, and the discovery requires methods of research and analysis appropriate to the particular level." [13]. 
It is this blending of three views on scientific analysis, a bottom-up view on knowledge integration, an inside-out view on knowledge organization, and a top-down view on knowledge validation, which engaged us to investigate integrative levels of organization as a more systematic approach to program comprehension. We have improved our own process of comprehension of intricate server-side components by combining the mindset of integrative levels with what psychology calls a cognitive feedback loop [10]. We approached program comprehension more systematically by studying the parts of a program in isolation, in terms that are meaningful to the end users and "just inclusive enough to have critical semantic mass" [14], as Quine put it; we documented and organized our program knowledge around a limited set of central ideas; we integrated the growing number of knowledge units into fewer yet semantically deeper units at a higher level of comprehension; and we fed back our emergent understanding into the process of acquiring improved grassroots understanding at the lower level.

The aim of this paper is to introduce the notion of integrative levels of organization into the landscape of program comprehension. We motivate our embracing of integrative levels by summarizing some of our own experiences in the arena of program maintenance and by discussing the conceptual shortfall of the pervading techniques of concept assignment. This sets the stage for a definition of the notion of an integrative level structure and for its application to program comprehension. We differentiate between extensional and intensional comprehension and define an intensional program level structure of five integrative levels the element level, the percept level, the concept level, the subject level, and the domain level. For evaluation purposes, we have organized the testing framework jUnit into such an intensional level structure.

\section{Research Motivation}

Our motivation to research integrative levels in the realm of program comprehension is primarily rooted in our day-to-day work of maintaining industrial programs, where we face tremendous costs for program comprehension. At the same time, we perceive dormant research activities that discuss more systematic and human-oriented approaches to program comprehension, of which most fall into the category of the concept assignment problem [3].

\subsection{Experiences in Program Comprehension}

Over the last decade, we have maintained distributed, server-side programs of large software systems in the telecommunications and graphic arts industries. In such environments, where the generations of program maintainers revolve rapidly, the pervading attitude towards program comprehension is still one which we like to portray as a puzzle of mental assembly of low-level program elements. The single most important tool of the program maintainers for the acquisition of knowledge about the structure of a program is the program debugger. The debugger helps them navigate through the relationships among the elements of the running program. Thus, they can investigate the syntactic neighborhoods around the elements encountered on their journey. They combine this information with pre-existing knowledge and information sought from external sources, such as peers and documents. Gradually, they start to adorn the low-level syntactic elements of a program with lowlevel semantic meaning, typically in terms of concepts that relate to the particular program maintenance task at hand. Repetitively yet progressively, they build up more encompassing and more abstract knowledge about these accumulated sets of program fragments. This is the way of program comprehension how we have experienced it and how others describe it [9].

Undeniably, a pragmatic stance on program comprehension has its benefits - after all you have to start the process of knowledge acquisition somewhere, and just enough knowledge is often good enough for the majority of corrective program maintenance tasks. Yet we have learned to repudiate this approach as a main reason that makes program maintainers almost always fall back into a more or less arbitrarily chosen form of implementation, before they even come close to a level of understanding of the application and technical requirements that a clean solution would demand. The fact is well-articulated that meaningful program comprehension, one that results in knowledge that can be explained in the terminology of the end users of the program, is hard if not impossible to achieve by employing a strict reductionist approach of navigation through low-level program elements. What is worse is that such a piecemeal strategy of program comprehension suppresses any genuine desire of program maintainers to rise above the technical aspects of a program and to understand its meaning for the end users. We have observed this to be a main reason for little motivation and devotion of many maintainers of large programs. 


\subsection{From Concept Assignment to Concept Integration}

Research in program comprehension has described many different techniques that aim at what Biggerstaff discussed as the concept assignment problem.

"Concept assignment is a process of recognizing concepts within a computer program and building up an "understanding" of the program by relating the recognized concepts to portions of the program, its operational context and to one another." [3]

Biggerstaff's description of the process of concept assignment as "a more opportunistic, nondeterministic and chaotic piecing together of evidence for a concept until some threshold of confidence is reached about its identity" [3] characterizes well its state of practice. There are many techniques that tackle concept assignment. Some of them are more syntactic, bottomup in nature, such as program slicing [19]; others employ a more semantic, top-down strategy, such as pattern-based reverse-engineering [7]. Our main concern with most techniques of concept assignment is that they attempt to inject from the outside some form of logic into a vast number of low-level program elements. This may be sufficient, sometimes, for the isolation of individual problems, but it is futile as an approach for the acquisition of deeper understanding about a complex, intricate program.

At the same time, however, we do not agree with a statement that concept assignment be intrinsically an opportunistic, nondeterministic, and chaotic process. We reflected about complex programs that we know well, how we managed to achieve a higher level of understanding, and how our understanding of such programs compares with other programs that we are uncomfortable maintaining. In sum, we believe that there is a process at play that enables us to comprehend the intricacies of a program faster and in less chaotic, more deterministic steps. This process operates in the form of integrative levels. In our research, we seek to improve the import of concept assignment with a process of progressive concept integration.

\section{The Integrative Level Structure}

In graph theory, a level structure is defined as a partition of a set of connected vertices into equivalence classes of vertices (i.e., the levels) with the same distance from a given root vertex, where the distance between two vertices is the number of edges in a shortest path connecting them. Figure 1 illustrates such a level structure with Units as the vertices and
Integration relations as the edges. This is what we refer to as an Integrative Level Structure.

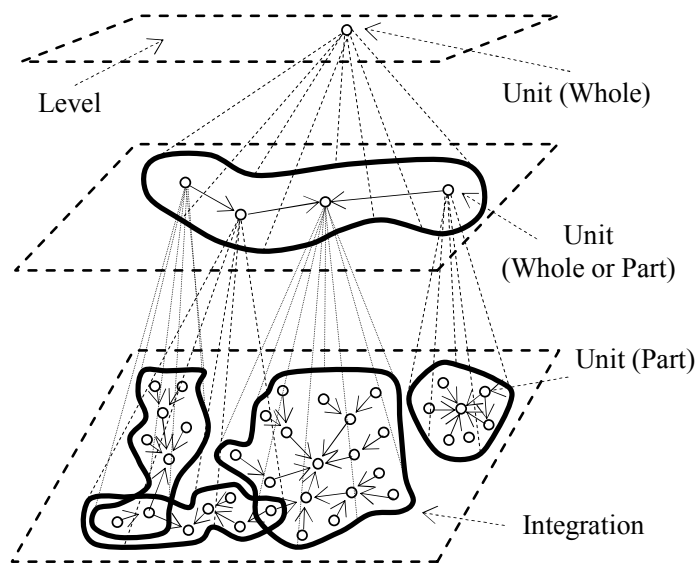

Figure 1: Definition of Integrative Level Structure.

It is the semantics of the integration relation which is at the heart of the Integrative Level Structure. By and large, the various usages of the notion of integration in literature have one characteristic in common: they treat integration as a dependence relation of strict partial order (i.e., irreflexive, antisymmetric, and transitive) among a set of Parts, one that gives name identity to the integrated Whole. As such, integration is often used ambiguously signifying element aggregation, component assembly, or organizational adaptation. Yet the meaning of integration is much deeper than an aggregation of individual Parts that form a lifeless Whole, as some pebbles may form a stone, or as often some program classes form a program package; neither is it a mere assembly-like sticking together of components; nor is it an effort to adapt systems to extend their organizational or functional reach. The very aim of integration is to tie a set of Parts into what Gestalt theory calls an organic Whole [11], one that has both structural and functional meaning as a Whole and one whose properties are perceived as more than the sum of its Parts. Such Wholes not only explain the order in which the Parts are combined, they also explain behavioural and dynamic aspects as superimposed over structural properties, and most importantly, they help explain, as Rescher and Oppenheim put it [15], such Whole properties that are not derivable from the individual Parts. These properties are said to have emerged through integration itself. Figure 2 illustrates the semantic aspects of the integration relation, which ties a set of individual Units at one level (i.e., the Parts) into exactly one Unit at the next higher level (i.e., the Whole). 


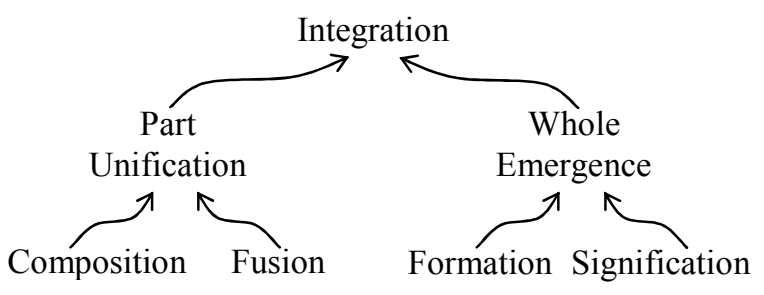

Figure 2: Integration Semantics.

Unification signifies a growing together of Parts into a Whole. The unification of Parts develops through Composition and Fusion. Composition describes the organizational order in which the Parts are unified. Although in practice, a composition often reflects a rather chaotic, arbitrary piecing together of Parts or a linear sequencing of Parts, Gestalt theory proposes that a natural and comprehensible composition of Parts develops from a central core outwards. Fusion describes the rules in which the Parts are amalgamated into a physically identifiable Whole, according to the prescribed compositional order.

Emergence signifies the appearance of such properties of the Whole that are not directly derivable from the sum of its Parts. These properties emerge from a unification of Parts through Formation and Signification. Formation describes the emergent structural and behavioural properties that form the Whole. These often obey to principles of domainspecific logic, which are concealed if one looks at the Whole as a mere aggregate of Parts. Signification describes the emergent meaning of Part properties within the context of the Whole.

\section{Integrative Levels of Program Comprehension}

Central to the theory of integrative levels of program comprehension is the transformation of a piecemeal, superficial structural explanation of a program into a more adequate structure for human comprehension. This is the goal for a definition of the integrative level structure of program comprehension.

In terms of logic, Carnap established that for every expression, there is the question of actual application and there is the question of meaning [4]. Hence there are two basic operations of comprehension. The first operation consists in investigating empirical situations referred to by the given expression and establishing factual truth. This is what Carnap calls understanding in extension. The second operation is a semantic one, which aims at grasping the meaning of an expression analytically. And this is what Carnap calls understanding in intension. It is the intension of an expression which allows us to discover the full extent of situations to which it applies. Therefore, Carnap concludes that "an expression has primarily an intension and secondarily an extension" [4]. The only clarification that we wish to add is that the intension of an expression is by necessity grounded in its extensional counterpart; hence there must be a shift in emphasis from extensional to intensional comprehension when progressing from early superficial understanding to later insightful understanding.

As a corollary to Carnap's conclusions, the goal of program comprehension is primarily one of understanding a program in intension and secondarily one of understanding it in extension. Only our understanding of the analytical meaning of a program, that is, its properties and its significance to the end users, will allow us to investigate its empirical, factual extent. Accordingly, Figure 3 differentiates between extensional and intensional program level structure.

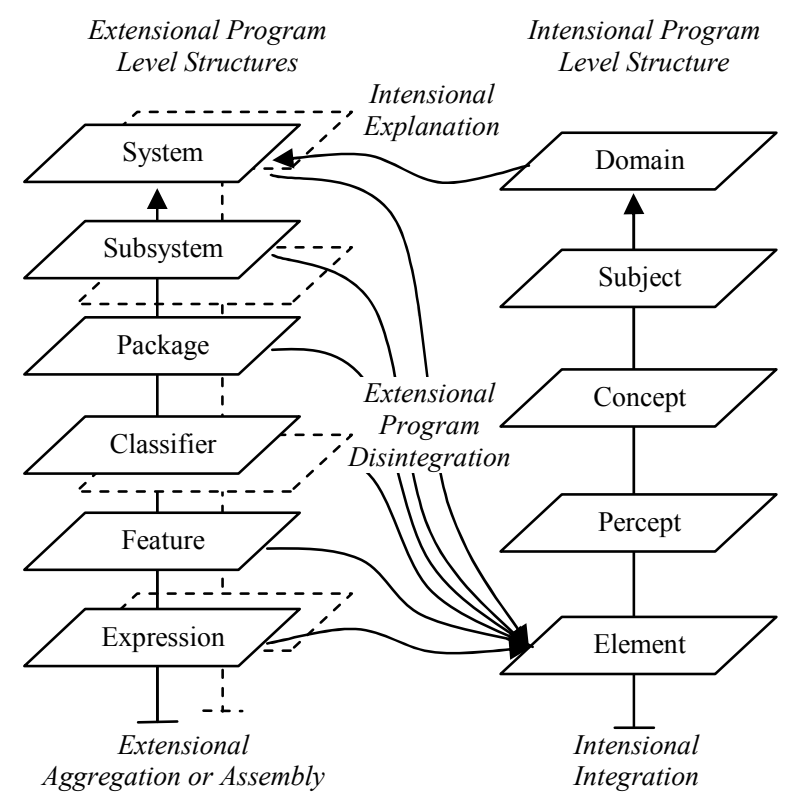

Figure 3: Extensional and Intensional Program Level Structures.

\subsection{The Extensional Program Level Structures}

The extensional level structures of a program reflect the physical composition of the program under study. Figure 3 illustrates a commonly used extensional level structure. Expressions build the ground level. Features, such as operations, methods, functions, and attributes, are integrated expressions. Classifiers integrate features into classes, interfaces, structures, and the like. Packages aggregate classifiers into macroscopic chunks, typically based on a coupling criterion. A Subsystem groups packages into independently 
evolving program clusters. A System aggregates a number of subsystems. This is likely the bestunderstood level structure of traditional program comprehension. Yet, from an extensional viewpoint, we must account for the fact that the observable extension of a program may well comprise more than one level structure. For instance, in larger programs, a second level structure accounts for the run-time execution. Such a level structure includes the levels Cluster, Node, Process, Thread Group, Thread, and Execution.

A main characteristic of the extensional level structures with which we are confronted in program comprehension is their being aggregative, assemblylike, rather than integrative. They often expose a superficial structural organization, which in based on certain technical infrastructure requirements. Although we acknowledge the importance of the extensional level structure for comprehension - after all they constitute the programming constructs that program maintainers look at in their day-to-day work - we do contend that the extensional level structures are of secondary importance. The main challenge of insightful program comprehension is to integrate the elements of the different extensional level structures into meaningful, intensional units that have significance in the program's end user domain and that can explain these higher-level program properties that are concealed by the technically biased organization of the extensional structure.

\subsection{The Intensional Program Level Structure}

There is only one intensional level structure. It is inherently integrative, in that it is formed in a process of part unification and whole emergence. It exposes those all-important program properties that are unknown to the extensional elements of the program in isolation. In the following, we briefly describe the five levels of the intensional program level structure.

An Element is an atomic unit that builds the lowest level of the intensional level structure. It is the constituent unit for what forms our early perceptions of the program. Elements are generated by the activity of disintegration of the extensional program level structures and by adorning the resultant sea of parts and part relationships with low-level semantics.

A Percept is the object of perception, a "mental impression of something perceived by the senses, viewed as the basic component in the formation of concepts" [1]. Percepts are formed by intensional integration of semantically related elements. They are the knowledge units that feed the generation of conceptual images of the program under study.
A Concept is "an idea of something formed by mentally combining all its characteristics or particulars" [5]. Concepts are the central knowledge units that link our lower-level percepts of the program with higher-level subjects.

A Subject is "a branch of knowledge" [5]. It constitutes a unit of study. Deep comprehension implies that one has absorbed the subject of study, such that one can explain the relationships of its concepts to other concepts of the same subject as well as their import to concepts of other subjects. A well-defined subject is free from incongruities and inconsistencies.

A Domain is "a realm of knowledge" [5]. Comprehension of a knowledge domain is only practicable if its subjects are loosely coupled. A domain is the intensional counterpart of the extensional system. The knowledge units that form the intensional level structure of the domain explain the knowledge units that form the extensional level structure of the system under study.

\section{The Process of Intensional Program Comprehension}

The start situation of intensional program comprehension is often perceived as being confronted with a pile of seemingly disconnected program elements that have little meaning to our common sense. The process of intensional program comprehension aims at integrating these low-level elements into higher-level units that are more appropriate for human comprehension. Its main objective is to uncover emergent program properties that are concealed in the organization of the extensional level structures. Emergent program properties arise from situations such as complex operation semantics, program extension logic, or performance-related features, to mention a few. These are often the source of the hard problems during program maintenance. The aspired end situation of intensional program comprehension is an intensional level structure whose units define such terminology that lends itself to effective explanations of intricate program properties.

The process of intensional program comprehension is inspired by Gestalt theory. In his acclaimed work on productive thinking, Wertheimer concludes with a list of knowledge operations that differentiate a productive thought process from commonplace processes which he describes as mechanical, reproductive, structurally blind, and piecemeal [17]. We amended Gestalt theoretical ideas with arguments of behavioral psychology. These hold that human thinking is driven in stimulus-response cycles that aim at a gradual reduction of incongruities in the comprehension of a 
state of affairs [10]. Based on our own experience in program maintenance, we reflected on how to apply these important studies of philosophy and psychology to program comprehension. We came up with an iterative process of intensional program comprehension that is based on the theory of integrative levels. Figure 4 gives a generic overview of this process.

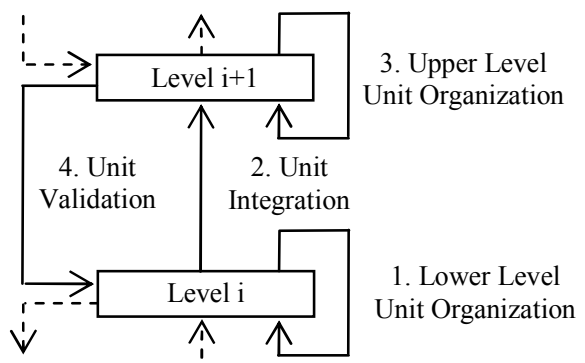

\section{Figure 4: Process of Intensional Program} Comprehension.

Every iteration of intensional program comprehension revolves around two adjacent levels (henceforth referred to as lower level and upper level) and it spans the four phases of lower level unit organization, unit integration, upper level unit organization, and unit validation. The following paragraphs outline typical activities of these four phases of a comprehension iteration.

1. Lower Level Unit Organization seeks comprehension of the units at a particular level in isolation and in relationship to other units at the same level. Typically, this phase is initiated in response to comprehension gaps and inconsistencies that were exposed during unit validation of the prior iteration:

- Maintain an intensional level diagram that illustrates intensional unit dependencies; organize intensionally peripheral units around more fundamental, central units.

- Keep the intensional import of the units of a level in balance; for example, do not promote what is clearly a lower-level Percept into a Concept.

2. Unit Integration seeks comprehension of the inner, intensional make up of an upper level unit, which includes the all-important emergent program properties that are unknown to the lower level units in isolation:

- Envisage a structural property of the program that is significant at a particular intensional level and can serve as a plan for integration.

- Recognize and investigate requirements and key scenarios that relate to that structural property.

- Identify intensional units at the next lower level that support this structural program property.
The above three more preparatory activities reflect a typical Unit Integration phase of a later iteration. At that stage of comprehension, Unit Integration is often driven by an idea about an upper level structural property that ripples down to the identification of lower level units supporting that idea. In contrast, in early iterations, when the conceptual structure of the program is still unclear, the contemplation of a structural property is more likely bottom-up, starting with the investigation of the extensional program level structure and the identification of requirements and key scenarios from which ideas for structural properties may be derived. The actual Unit Integration activities touch upon the very semantics of Integration as discussed in Figure 2:

- Identify or create an upper level unit with a name that implies the property being looked at; assign the property to this upper level unit.

- Illustrate the composition dependencies of the identified lower level units in a separate diagram.

- Illustrate the fusion of the lower level units in a separate diagram.

- Describe the formation of emergent properties of the higher level unit, for instance, by annotation of the fusion diagram.

- Capture the emerging signification of lower level units as a part of the higher level unit.

3. Upper Level Unit Organization seeks comprehension of the fitness of an emergent unit in the broader picture of the higher level:

- Incorporate units that emerge by integration into the intensional level diagram of the upper level; identify their intensional location and their dependencies to other units at the upper level.

- Follow up on the formation and signification of emergent properties and see how they change the meaning of other units at the same level.

A semantic change to a unit at the upper level always sparks off a comprehension iteration that starts at the upper level. Thus, intensional program comprehension often spirals upwards to the top level.

4. Unit Validation seeks to spot gaps, incongruities, and superficialities in the comprehension of a unit:

- Justify the criterion of a well-formed Gestalt: "the parts are determined by the structure of the whole, as the whole is by the parts" [17].

- Write tests that verify the intension of the unit against the executable program.

Unit validation often generates another comprehension iteration that aims at closing any discovered comprehension gaps. 


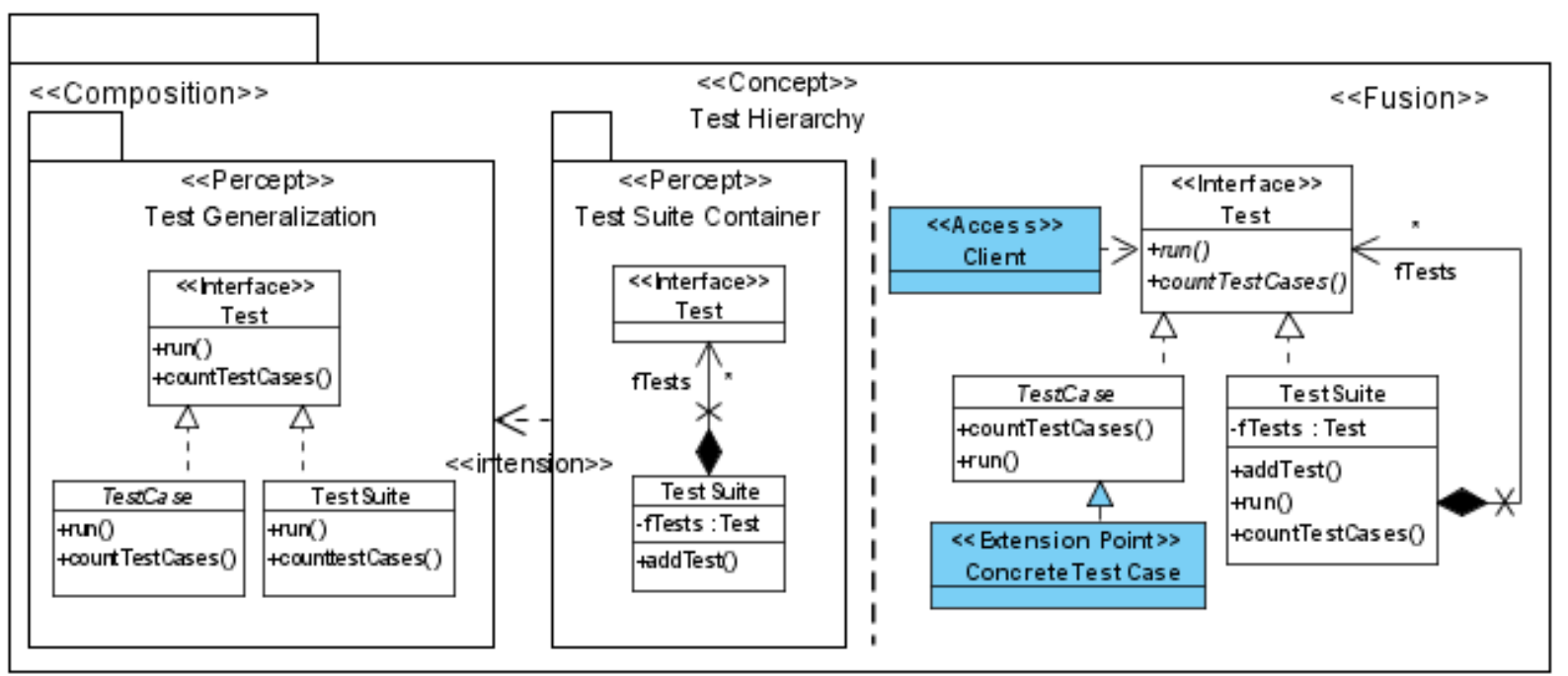

Figure 5: Test Hierarchy Concept: Composition Diagram (left part) and Fusion Diagram (right part).

\section{The jUnit Case Study}

We chose the testing framework $j$ Unit 4.4 (hereafter referred to as $j U n i t)$ for the discussion of integrative levels of program comprehension. It has a limited scope, yet it is complex enough to communicate all major issues. $j$ Unit was designed as a composition of design patterns, thus allowing for many intricate ways of extension. In one of our industrial projects, we extended $j$ Unit to drive a test harness from proprietary XML-based test case specifications. During this exercise, we realized that this program is much more subtle than the size of its source code might imply.

We used Visual Paradigm for UML 6.3 to reverseengineer class and package diagrams from the source code of $j$ Unit. In the following, we summarize some typical comprehension activities that we performed in the process of building up an intensional program level structure of $j$ Unit. The objective of this section is not to provide a detailed explanation of jUnit. Rather, our intent is to illustrate the importance of integrative, intensional program comprehension by applying a few of its activities to a concrete case study.

Activity 1: Low Level Element Organization. To obtain an overview of the size, style, and organization of $j$ Unit, we walked through the packages and classes, we annotated relationships with aggregation semantics, visibility, multiplicity, and navigability, we improved the diagram layout, and we added stereotypes as indicators of threading, coordination, and interfacing. The aim of this initial activity was to improve the organization of the element level for human comprehension. During its course, we became acquainted with the programming style and the programming culture in which $j$ Unit was crafted.
Activity 2: Element Integration (Unification). As we developed an idea of the comprehension task ahead, we identified small sets of elements with strong semantic relationships. In framework-like programs, such relationships are often based on generalization and aggregation. For example, by visual inspection of the $j$ Unit element level, we could easily fathom the meaning of the base classes Test, TestCase, and TestSuite of its main class hierarchy. As we restructured the layout of the model, the importance of a recursive aggregation relationship between TestSuite and its own interface Test became very noticeable. We decided to elevate the semantic relationships among these elements and unify them into the Test Generalization Percept and the Test Suite Container Percept, respectively (Figure 5, left part).

We integrated many more such elements into percepts. We started some iterations in parallel, but we always completed every iteration with an examination of the validity of the integrated percept. In most cases, this involved several iterations in which we adjusted the lower level element organization, the integration semantics of the percepts, and the upper level percept organization. With a growing picture of low-level program semantics, percept integration became more intuitive and lost its non-deterministic, chaotic character as described by Biggerstaff [3].

Activity 3: Percept Integration (Unification and Formation). In many percept level iterations, often interleaved with lower element level iterations, we unified the growing number of percepts into a much smaller set of concepts, yet with deeper intensional meanings. Our knowledge of design patterns guided the formation of the unified percepts. Yet our approach was not one of assigning the prescribed roles of a design pattern to extensional program classes; rather, 


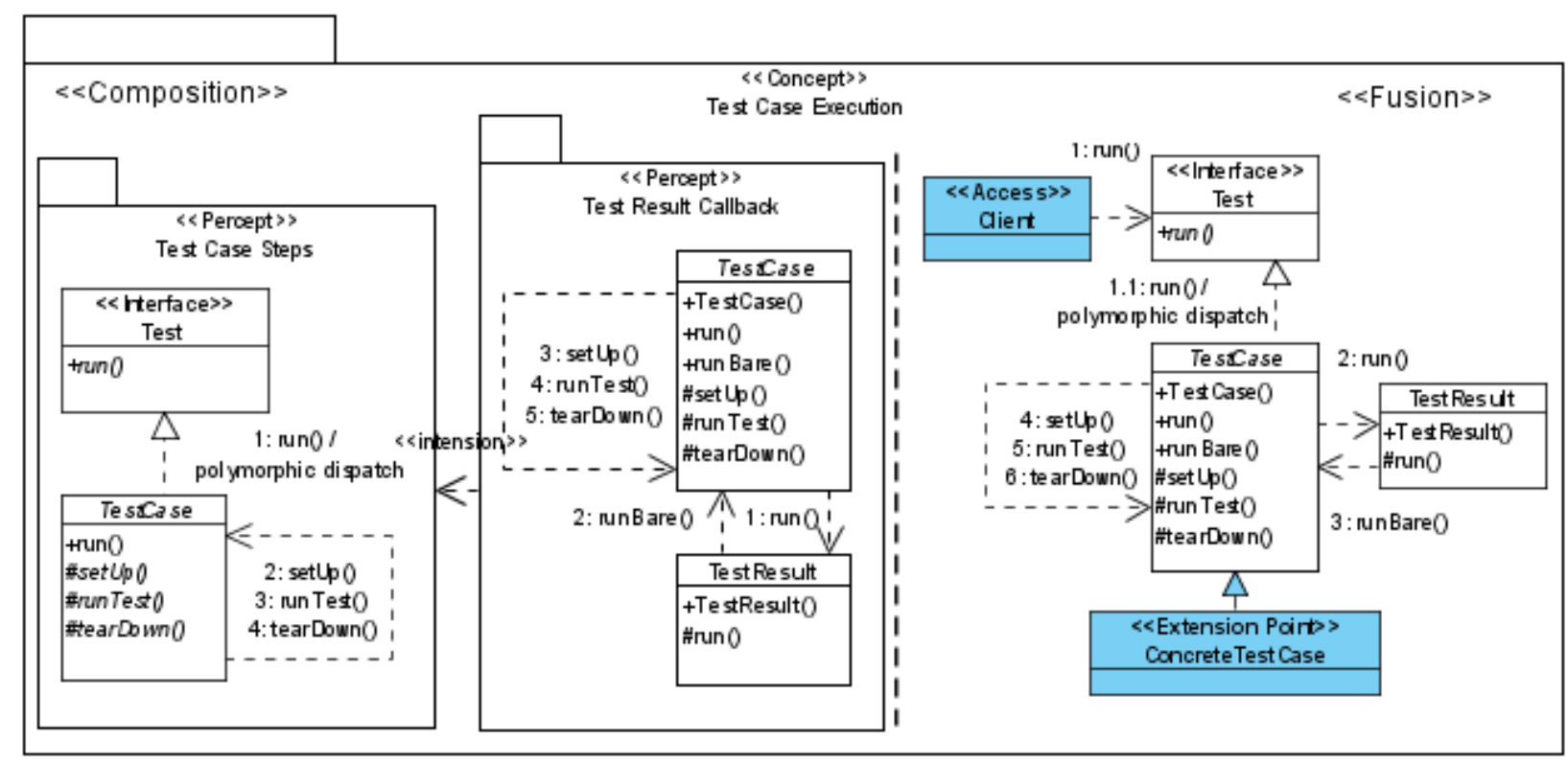

Figure 6: Test Case Execution Concept: Composition Diagram (left part); Fusion Diagram (right part).

we used the design pattern to justify the unification of percepts. In other words, the design pattern laid out a course plan [10] for the formation of a concept via the unification of percepts. The unification activity itself was still driven bottom-up, by understanding how the intension of the percept fit into the formation plan of the concept. For example, in Figure 5 we used the Composite design pattern as a plan for the unification of the Test Generalization Percept and the Test Suite Container Percept into the Test Hierarchy Concept.

In more intricate, industrial programs, such a priori design knowledge is often not available. In these situations, the identification and consolidation of a structural plan for percept integration takes many more iterations of comprehension.

Activity 4: Concept Validation Stimulating Changes to a Percept Integration. We continued with a more thorough examination of the semantics of the Test Hierarchy Concept against the extensional level structure. It struck us that the mechanism for the creation of test suites was quite different from our understanding of the Composite design. The TestSuite class of $j$ Unit uses a reflective mechanism to instantiate TestCase objects. Understanding of this mechanism is crucial for the proper use of $j U n i t$, and so we decided to create a Test Suite Creation Percept covering this knowledge. In a subsequent iteration we unified this percept with the other percepts of the Test Hierarchy Concept.

Above explication of the activities that led us to the Test Suite Creation Percept and its unification into the Test Hierarchy Concept is a good example for what we refer to as a comprehension iteration. Our desire to find structural integrity in jUnit led us to validate the intension of the Test Hierarchy Concept, which revealed gaps in our understanding of some of its parts. This stimulated changes to the organization of the lower percept and element levels, the integration of those elements that caused our bewilderment into a new Test Suite Creation Percept, the alteration of the integration semantics of the existing Test Hierarchy Concept, and the repositioning of the Test Hierarchy Concept within the intensional level diagram of the Concept level. Intensional program comprehension works in many such cycles. Oftentimes, they are interleaved by comprehension cycles at lower or higher levels, but they always follow the iterative comprehension model as illustrated in Figure 4.

Activity 5: Element Integrations. We continued in our quest to acquire knowledge about jUnit by removing as many incongruities in our understanding as possible, which certainly included the test execution mechanism. We started our program debugger and walked through the execution graph of a typical test case. The debugger steered us into the TestCase class and revealed a complex invocation sequence that resembled a combination of a template method whose hook methods were invoked via a callback mechanism from a class TestResult. The rationale for this indirection was not clear to us at all, but our feel about the importance of the underlying design led us to integrate the involved elements into two new percepts, the Test Case Steps Percept and the Test Result Callback Percept. After a short period of reflection, we decided to amend both percepts with invocation 
semantics such that it can facilitate subsequent rounds of comprehension. Figure 6 illustrates the two percepts.

Activity 6: Percept and Concept Organization. As discussed in the introduction, Gestalt theory suggests that our perceptions of reality are organized in a hierarchical form around a central point [8]. Our experiences in program comprehension confirm that such an inside-out view significantly enhances our cognitive capabilities. To reinforce inside-out comprehension, we sought an intensional order among the units at higher intensional levels. shows the intensional order among the percepts that we discussed above. The importance of the intensional dependency diagram is that it provides a clear direction of program comprehension, which is from an essential intensional core outwards into more peripheral facets.

Activity 7: Percept Integration (Signification). As an example of an emergent signification, we use the run operation of the Test interface. At the Element level, the semantics of the run operation refers to many seemingly intensionally detached elements. Meaning of the run operation only arises in the bigger pictures of the four percepts of Figure 5 and Figure 6. These images clearly imply observable program properties, which includes a uniform access to both test cases and test suites (Test Generalization Percept), a recursive execution of all tests of a test suite (Test Suite Container), a test execution in three steps for the setup, the actual run, and the tearing down of the test (Test Steps Percept), and the collection of the result of each test case execution (Test Result Callback). Yet only the integrated concepts, the Test Hierarchy Concept and the Test Case Execution Concept, have the critical semantic mass from which program maintainers can imply higher-level program properties, which are, at best, only superficially discernible at the lower levels. As an example, we annotated the fusion diagram in Figure 6 with a stereotyped ConcreteTestCase class which signifies how to extend a test hierarchy with concrete test run implementations. Such program extension logic is often only available or meaningful at the concept level. In more industrial programs, important emergent properties are related to performance and stability.

Activity 8: Intensional Program Comprehension Cycle. In the course of our study, we entered an iterative and incremental knowledge acquisition cycle of percept organization, percept integration, concept organization, concept validation, and back to percept organization. We supported these intensional comprehension activities with extensional activities of program inspection and runtime program navigation. We removed many of the incongruities in our understanding of $j$ Unit. Some were related to exception handling, test result collection, test observation, and test decoration. Gradually, we organized the allimportant concept level of the intensional level structure of jUnit. Most important, in the end we had a trace of the comprehension process in form of concepts, percepts, and elements. This enabled us to transfer our knowledge of $j$ Unit to others on the team more effectively.

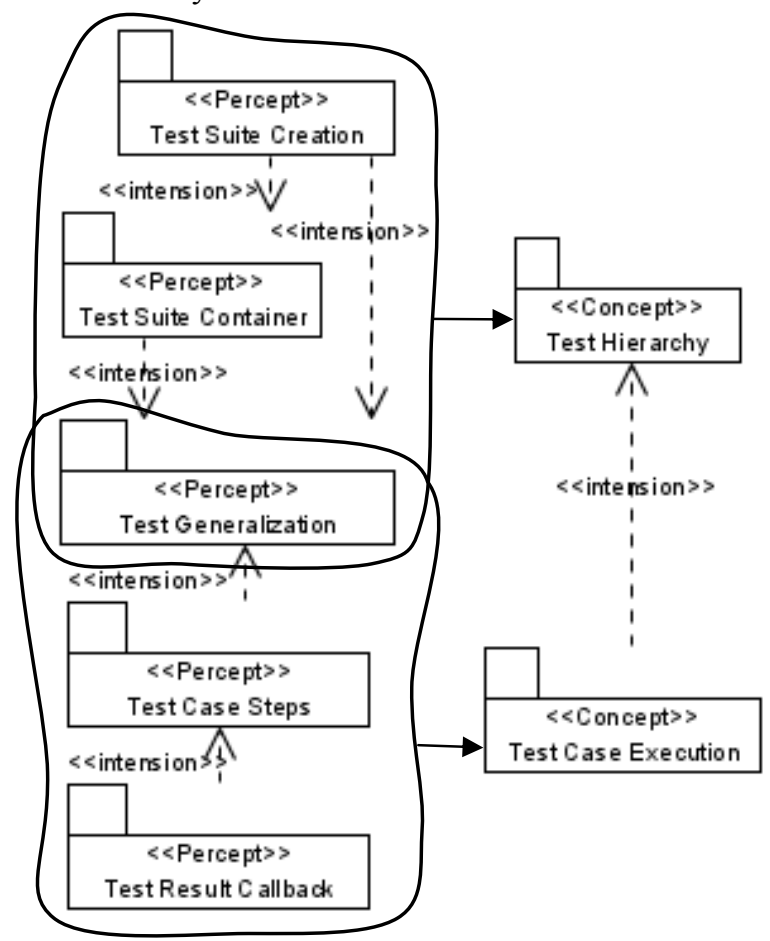

Figure 7: Intensional Organization of Integrated Percepts.

\section{Conclusion}

In this paper, we discussed and applied integrative levels of organization to program comprehension. We based program comprehension on Gestalt theoretical hypotheses and postulated that (a) productive program comprehension is not a piecemeal, assembly-like activity, but rather demands a prior structural plan of the confronted situation and an investigation how the observable parts of this situation fit into this plan and (b) that productive program comprehension grows at different levels around a small conceptual core.

We followed the work of Carnap on meaning [4] and proposed that program comprehension faces two kinds of level structures: (a) the extensional level structures, which replicate the observable structures of the program and (b) an intensional level structure, which reflects our understanding of the meaning of the program. We concluded that program comprehension is a process that is primarily concerned with intension and secondarily with extension, which stands in stark 
contrast to our practical experiences in program maintenance.

We detailed the semantics of integration, defined, five levels of integrative, intensional program comprehension, and outlined an iterative process for the transformation of the aggregative-structured, technically-biased extensional level structures into an integrative-structured, human-oriented intensional level structure. Based on the jUnit case study, we worked out the greatest strength of integrative level of program comprehension, which is its capability to help uncover higher-level program properties that are beyond the reach of the extensional structures. The theory of integrative levels refers to such properties as emergent properties. In fact, a disciplined application of integrative levels of program comprehension often steers the program analyst to their discovery, which is for us the most important finding.

\section{Future Work}

As part of our practical work, we are in the process of transforming the extensional level structures of a typical industrial software component, which is CORBA-based, multi-threaded, database-driven, performance-enhanced with in-memory caches, and exception intensive, into an intensional level structure. This will help further study of the semantics of intensional integration and the general characteristics of the units that build the various levels of the intensional program level structure.

As part of our academic research, we are working on the formalization of the integrative, intensional program level structure. This will provide a basis for tool-supported integrative comprehension techniques. Furthermore, we plan to discuss the nature of efficient explanations of complex program properties on the ground of integrative levels. Finally, we intend to work on the extraction of intensional units at the concept level such that the validation of the intension against the extension may be possible.

\section{References}

[1] The American Heritage Dictionary of the English Language, Fourth Edition. From Dictionary.com website.

[2] P. W. Anderson. More is Different. In Science, Vol. 177, No. 4047, August 1972.
[3] T. J. Biggerstaff. The Concept Assignment Problem in Program Understanding. In Proc. of the $13^{\text {th }}$ International Conference on Software Engineering, Baltimore, Maryland, USA, May 1993, pp. 482-498.

[4] R. Carnap. Meaning and Necessity: A Study in Semantics and Model Logic. The University of Chicago Press, Chicago, USA, 1956.

[5] Dictionary.com Unabridged (v 1.1$)$. From Dictionary.com website.

[6] J. Feibleman. Theory of Integrative Levels. In The British Journal for the Philosophy of Science, Vol. 5, No. 17, May 1954, pp. 59- 66.

[7] R. K. Keller, R. Schauer, S. Robitaille, and P. Pagé. Pattern-Based Reverse-Engineering of Design Components. In Proc. of the $21^{\text {st }}$ International Conference on Software Engineering, Los Angeles, CA, USA, May 1999, pp. 226-235.

[8] D. B. King and M. Wertheimer. Max Wertheimer \& Gestalt Theory. Transaction Publishers, New Jersey, USA, 2005.

[9] A. Mayrhauser and A. M. Vans. Program Comprehension during Software Maintenance and Evolution. IEEE Computer, Vol. 28, No. 8, 1995, pp. 44-55.

[10] G. Miller, E. Galanter, and K. H. Pribram. Plans and the Structure of Behavior. Henry Holt and Company, New York, USA, 1959.

[11] E. Nagel. Wholes, Sums, and Organic Unities. In Philosophical Studies, Eds. H. Feigl and W. Sellars, Vol. 3, No. 2, 1952, pp. 17-32.

[12] J. Needham. Integrative Levels: A Revaluation of the Idea of Progress. Clarendon Press, Oxford, 1937.

[13] A. Novikoff. The Concept of Integrative Levels and Biology. Science, Vol. 101, 1945.

[14] W. V. Quine. Two Dogmas in Retrospect. In Canadian Journal of Philosophy, Vol. 21, No. 3, September 1991.

[15] N. Rescher and P. Oppenheim. Logical Analysis of Gestalt Concepts. In The British Journal for the Philosophy of Science, Vol. 6, No. 22, August 1955, pp. 89- 106.

[16] T. C. Schneirla. A Consideration of Some Conceptual Trends in Comparative Psychology. In Psychological Bulletin, Vol. 49, 1952, pp. 559-597.

[17] M. Wertheimer. Productive Thinking. Tavistock Publication, London, U.K., 1959.

[18] M. Wertheimer. Gestalt Theory. In W. E. Ellis (Ed.), A Source Book of Gestalt Psychology, Routledge \& Kegan Paul, Trench, Trubner \& Co., Ltd., London, UK, 1938.

[19] M. Weiser. Program slicing. IEEE Transactions on Software Engineering, Vol 10, No. 4, July 1984, pp. 352-357. 